\title{
Research on Influencing Factors of Usage Intension to Use Mobile Intelligent Wearable Device
}

\author{
Huijun Zhou ${ }^{1}$ and Jihkuang Chen ${ }^{2, b^{*}}$ \\ ${ }^{1,2}$ School of Management, Xiamen University Tan Kah Kee College \\ bpony1146@163.com
}

Keywords: Mobile Intelligent Wearable Device; UTAUT2; DEMATEL

\begin{abstract}
Although the rise of mobile intelligent wearable devices, however it is still in the primary stage and consumers have some doubts about its network stability and device stability. Therefore, what factors may affect the willingness and behavior of consumers to wear mobile intelligent devices will become the key problem to be solved by mobile intelligent wearable device manufacturers. This study cites a decision making and evaluation laboratory (DEMATEL) to comprehensively analyze the influence between factors. The advantage is that not only a small amount of samples are needed, but also the influence degree between the factors can be analyzed from the influence relationship between the factors, thereby obtaining the management decision direction.
\end{abstract}

\section{Introduction}

In recent years, with the maturity of computer and Internet technology, the rise of mobile intelligent wearable devices makes intelligent devices closer to consumers and more portable and compact, it also plays a good media role in analyzing and calculating all aspects of human body data or providing external conditions. As a result, mobile intelligent wearable devices are widely used in many fields such as medical health, sports outdoor, leisure and entertainment. However, because the mobile intelligent wearable device is still in the primary stage, the R \& D of its software and hardware technology is not mature enough, and consumers have some doubts about its network stability and device stability. Therefore, what factors may affect the willingness and behavior of consumers to wear mobile intelligent devices will become the key problem to be solved by mobile intelligent wearable device manufacturers.

With the rapid development of academic research on the acceptance of new technologies, there are increasing relevant theoretical models. Venkatesh et al. (2003) [1] found that the theoretical models of technology acceptance proposed by past studies have their corresponding explanatory powers in their respective fields. Therefore, they integrated these theoretical models and proposed an integrated technology acceptance model (UTAUT model). In 2012, this model was extended to propose a more complete UTAUT2 model (Venkatesh et al. 2012) [2]. In the past, some studies have proved that the theoretical model can be applied in many fields and can effectively explain the process of consumers' acceptance and adoption of new technologies [3-5]. Relevant research also confirmed the applicability of the UTAUT model in studying the willingness to use and the influencing factors of users of mobile intelligent wearable devices [6-7], however, there are few studies in the UTAUT2 model, and analyzed by statistical technique.

However, Lee et al. (2010) [8] pointed out that two possible shortcomings in measuring the influence of various factors by statistical techniques such as regression testing or structural equation modeling: I. A large number of questionnaires have to be conducted to target groups, which is time-consuming and laborious; II. Based on the existing architecture of the model, the possible interaction between factors cannot be fully considered, and the exact results may not be obtained. Therefore, this study cites a decision making and evaluation laboratory (DEMATEL) to comprehensively analyze the influence between factors. The advantage is that not only a small amount of samples are needed, but also the influence degree between the factors can be analyzed from the influence relationship between the factors, thereby obtaining the management decision direction. 


\section{Method}

UTAUT2 model. In UTAUT2 model, the seven core including Performance Expectancy; Effort Expectancy; Social Influence; Facilitating Conditions; Hedonic Motivation; Price Value and Habit, determinants that play a significant role as direct determinants of Behavior Intension and Usage Behavior, meanwhile Usage Behavior will be affected by Facilitating Conditions and Habit.

\section{DEMATEL technique.}

(1) Establish direct-relation matrix

With the opinion integration of all experts, the direct-relation matrix, $X$, of $n \times n$ can be obtained. In the direct relation matrix, $X, x_{i j}$ represents the variable, the level of $i$ affects variable $j$, and the diagonal variable $\mathrm{x}_{\mathrm{ij}}$ of the direct-relation matrix, $\mathrm{X}$ is set to 0 .

(2) Calculate normalized direct-relation matrix

The method of normalized direct-relation matrixes $\mathrm{N}$, uses the biggest sum of the row vector as the normalized base.

(3) Calculate total-relation matrix

The total-relation matrix, $\mathrm{T}$, can be obtained from Eq. (1)

$$
\mathrm{T}=\operatorname{limit}_{\mathrm{w} \rightarrow \infty}\left(N+N^{2}+\ldots \ldots+N^{w}\right)=N(I-N)^{-1}
$$

(4) Calculate the total influence degree of each variable and the degree of being influenced

Set $t_{i j}$ as the variable of the total-relation matrix, $\mathrm{T}$, and $\mathrm{i}, \mathrm{j}=1,2, \ldots, \mathrm{n}$. The total influence degree of each variable and the degree of being influenced can be calculated from Eqs. (4). Set $\mathrm{D}_{\mathrm{i}}$ as the sum of row $i$, which represents the variable $i$, and is the cause that affects the sum of other variables. $R_{j}$ is the sum of column $j$, which represents the variable $j$, and is the result and the sum of affected by other variables. $D_{i}$ and $R_{j}$ includes direct and indirect influences.

$$
D_{i}=\sum_{j=1}^{n} t_{i j}(i=1,2, \ldots ., n), R j=\sum_{j=1}^{n} t_{i j}(j=1,2, \ldots ., n),
$$

(5) Calculate the prominence and relation of all variables

Define $\left(D_{k}+R_{k}\right)$ as the Prominence, and $k=i=j=1,2, \ldots, n$, which shows the overall level of this variable being affected and its influence on others. This score shows the core level of variable $\mathrm{k}$ in this case; and $\left(D_{k}-R_{k}\right)$ is defined as the Relation, which means the gap level of the variable being affected and its influence on others.

\section{Case Study}

Model Establishment. Based on the UTAUT2 model, this study introduces Fashion Perception variables, which refer to consumers' perceptions of the novelty, entertainment and trend of new technologies [10]. According to the research purpose, in order to make the mobile intelligent wearable device more compatible with this theoretical model, this study uses Entertainment Perception to measure the concept of hedonic motivation in the theoretical model, and uses Value Perception to measure the concept of price value in the theoretical model. In addition, as a result of interviews with small sample groups, a larger proportion of users wearing mobile intelligent devices are less affected by the population around them. Therefore, this study removes the Social Influence from the original model.

Questionnaire Design and Investigation. This study made 9 pairs of influence factors questionnaires in 9 factors in UTAUT2. A total of 30 senior users with 2 years or more of experience in wearable device use were selected from the mobile intelligent wearable device users group (user age group is between 20-45 years old, 17 female users, 13 male users). After collecting the opinions of 30 sample objects by interview and questionnaire, the direct relationship matrix was merged and analyzed by DEMATEL.

\section{DEMATEL Analysis.}

(1) Direct Relation Matrix

After interviewing with 30 sample subjects, the scores of the nine sample subjects were averaged between the nine factors, and the initial matrix can be obtained. 
(2) Total Relation Matrix

After the initial direct relation matrix is obtained, the normalized direct relation matrix is calculated according to the biggest sum of the row vector $\lambda=0.10$. Then the direct relation matrix $X$ is multiplied by the $\lambda$ value, and the normalized direct relation matrix $\mathrm{N}$ is obtained. Then, according to formula (1), the total relation matrix $\mathrm{T}$ is calculated. The results are shown in the following, where, for simplified analysis, those with smaller impact values are discarded (the cutoff value is set to 0.282 ):

\begin{tabular}{|c|c|c|c|c|c|c|c|c|c|}
\hline$C U T$ OFF $=0.282$ & s1 & 52 & 53 & $x 4$ & $x 5$ & 56 & $x 7$ & 58 & is \\
\hline $\mathbf{x 1}$ & 0 & 0 & 0 & 0,284 & 0 & 0 & 0 & 0.520 & 0 \\
\hline $\mathrm{s} 2^{2}-1$ & 0 & 0 & 0 & 0 & 0 & 0 & 0 & 0.409 & 0 \\
\hline 83 & 0 & 0 & 0 & 0.285 & 0 & 0 & 0 & 0.572 & $0.4 \pi$ \\
\hline 34 & 0 & 0 & 0 & 0 & 0 & 0 & 0 & 0.607 & 0 \\
\hline$x 5$ & 0 & 0 & 0 & 0 & 0 & 0 & 0 & 0.403 & 0 \\
\hline *6 & 0 & 0 & 0 & 0 & 0 & 0 & 0 & 0,418 & $0+12$ \\
\hline$x 7$ & 0 & 0 & 0 & 0 & 0 & 0.362 & 0 & 0.560 & 0.558 \\
\hline xs & 0 & 0 & 0 & 0 & 0 & 0 & 0 & 0 & 0.339 \\
\hline$x 9$ & 0 & 0 & 0 & 0 & 0 & 0 & 0 & 0 & 0 \\
\hline
\end{tabular}

(3) Influence Degree Analysis

According to the total relation matrix, the influence degree can be obtained. According to the architecture of UTAUT2, the complete architecture of the influencing factors of the mobile intelligent wearable device usage intension to use is re-established, as shown in the Figure 1:

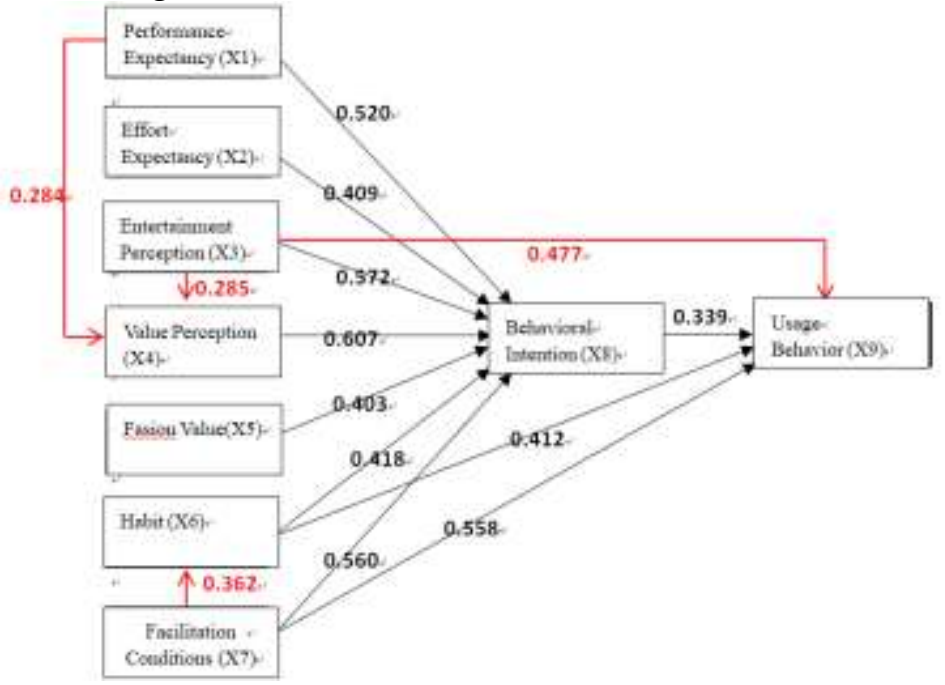

Figure 1. Factor influence diagram

Discussion. From the Figure 1, it is found that the four newly added factors do not exist in the original UTAUT2 model. The discussion is as follows:

(1) X3 Entertainment Perception has a great influence on X4 Value Perception, and the weight is 0.285 , which is positive correlation. That is, the higher the perceived sensation perceived by the consumer using the intelligent wearable device, the more the value of using the intelligent wearable device to make the individual feel polite, and thus the sensitivity to the cost is lower. In addition, X3 Entertainment Perception has a great influence on X9 Usage Behavior with a weight of 0.477 and a positive correlation. That is, when consumers use a certain intelligent wearable device, the higher the sense of pleasure, the higher the acceptance and the willingness to try for the similar or similar intelligent products, thus directly produce the use behavior to obtain the sense of pleasure.

(2) X1 Performance Expectation has a great influence on X4 Value Perception, and the weight is 0.284 , and it is positive correlation. That is, the more obvious the performance improvement that consumers perceive when they use intelligent wearable devices, the more they can feel the value of using intelligent wearable devices to improve their personal lives and work efficiency, so as to improve the value of the product perception.

(3) X7 Facilitation Conditions has a greater impact on the X6 Habits, and the weight is 0.362 , and is positively correlated. That is, when consumers perceive the convenience and various technical conditions of intelligent wearable devices to be complete, it is more helpful to promote the stability preference of users, and thus to keep the willingness and behavior of using this kind of 
devices.

Causality Diagram Analysis. Di and $\mathrm{Rj}$ and the degree of center (Di+Rj) and reason (Di-Rj) are calculated according to the complete relation matrix. The results are shown in following Table 1:

\begin{tabular}{|c|c|c|c|c|}
\hline & Di & $\mathrm{Ri}$ & $\mathrm{D}+\mathrm{R}$ & D-R \\
\hline $\mathbf{x} 1$ & 0.804 & 0.000 & 0.804 & 0.804 \\
\hline$x 2$ & 0.409 & 0.000 & 0.409 & 0.409 \\
\hline x3 & 1. 334 & 0.000 & 1. 334 & 1. 334 \\
\hline$\times 4$ & 0.607 & 0.569 & 1. 176 & 0.038 \\
\hline x5 & 0.403 & 0.000 & 0.403 & 0.403 \\
\hline$x 6$ & 0.830 & 0.362 & 1. 192 & 0.468 \\
\hline$x 7$ & 1. 479 & 0.000 & 1. 479 & 1. 479 \\
\hline$x 8$ & 0.339 & 3. 488 & 3.827 & -3.150 \\
\hline x9 & 0.000 & 1. 786 & 1. 786 & -1.786 \\
\hline
\end{tabular}

Furthermore, a causal diagram is drawn based on the calculation of centrality and causality, as shown in Figure 2 below:

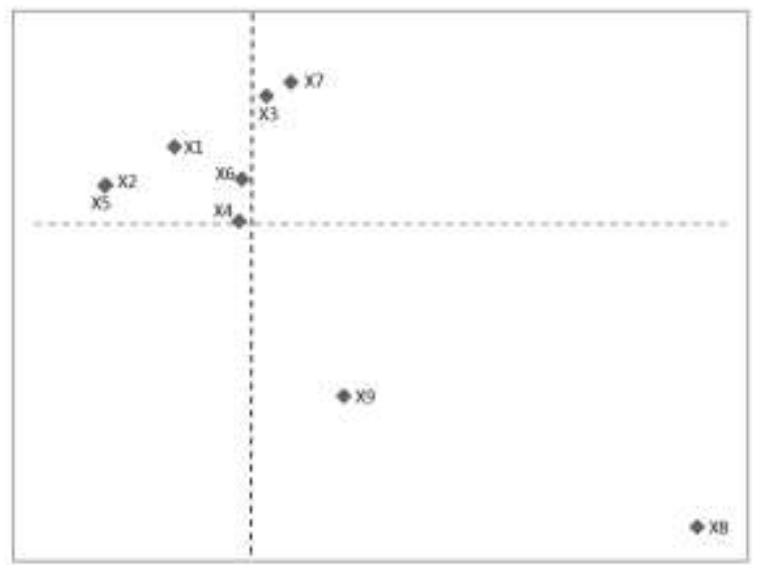

Figure 2. Causality diagram

Result. In view of the above results, the important influencing factors in the core improvement area are the X3 entertainment perception and the X7 promotion condition, respectively. In order to promote the overall acceptance of mobile intelligent wearable devices, priority should be given to driving factors, including improvements in X1 performance expectations, X2 effort expectations, $\mathrm{X} 4$ value perception, $\mathrm{X} 5$ fashion perception, and X6 usage habits.

\section{Conclusion}

This study applied the DEMATEL method to explore the causal relationship between the nine influencing factors that may affect the user's willingness to use and behavior of mobile smart wearable devices, re-constructed the UTAUT2 technology acceptance model, which is more representative of users' willingness to use mobile smart wearable devices, and obtained the direction of improvement and management decision-making. It assists equipment manufacturers to better improve the functions and services of mobile smart wearable devices, and provides reference for equipment manufacturers in product design and manufacturing, marketing strategy formulation, and service level improvement. However, there are still some defects in this study, such as the lack of representativeness and extensiveness of the samples, and the integration and discussion of the influencing factors. Besides, the method of synthesizing the opinions of sample objects can also be integrated with fuzzy theory to enhance the rigor of the research, which is the direction that can be further strengthened in the future research.

\section{References}

[1] V. Venkatesh, M.G, Morris, G.B, Davis et al, User Acceptance of Information Technology: 
Toward a Unified View, MIS Quarterly, 27 (2003) 425-478.

[2] V. Venkatesh, J.Y.L Thong, and $\mathrm{X}$. Xu, Consumer Acceptance and Use of Information Technology: Extending the Unified Theory of Acceptance and Use of Technology, Social Science Electronic Publishing, 36 (2012) 157-178.

[3] L.H. Lvi, and Y. Chen, Research on the Influencing Factors of Initial Trust of Booking Travel APP Users, Seeker, 10 (2016) 112-117.

[4] M. Zhang, and S. Lin, Research on the Influencing Factors of College Students' Use Behavior of Instant Messaging Software, Shanghai Management Science, 38 (2016) 66-70.

[5] J. Lan, and D.J. Zhu, Research on Acceptance and Use Behavior of Sustainable Transportation Consumption-Based on the Investigation of Shanghai Automobile Sharing, China Population, Resources and Environment, 26 (2016) 98-105.

[6] J. Ma, and Z.P. Dong, Empirical Research on Consumer Acceptance of Wearable Devices, Future and Development, 9 (2015) 37-41.

[7] Z.W. Gu, F.Y. Xu, and J. Wei, An Empirical Study on the Influencing Factors of the Initial Trust of Wearable Business Consumers, Management Review, 27 (2015) 168-176.

[8] Y.C. Lee, M.L. Li, T.M. YEN, and T.H. Huang, Analysis of adopting an integrated decision making trial and evaluation laboratory on a technology acceptance model, Expert Systems with Applications, 37 (2010) 1745-1754.

[9] A. Gabus and E. Fontela, Perceptions of the world problematique: Communication procedure, communicating with those bearing collective responsibility. DEMATEL Report No. 1, Battelle Geneva Research Center, Geneva, Switzerland, 1973.

[10]Z. Yang, Research on Factors Affecting User Acceptance of Wearable Devices, Beijing University of Posts and Telecommunications, 2015. 